(c) American Dairy Science Association, 2004.

\title{
The Distribution of Mycobacterium avium ssp. paratuberculosis in the Environment Surrounding Minnesota Dairy Farms
}

\author{
E. A. Raizman, ${ }^{1}$ S. J Wells, ${ }^{1}$ S. M. Godden, ${ }^{1}$ R. F. Bey, ${ }^{2}$ \\ M. J. Oakes, ${ }^{3}$ D. C. Bentley, ${ }^{4}$ and K. E. Olsen ${ }^{4}$ \\ ${ }^{1}$ Department of Veterinary Population Medicine, \\ 2Department of Pathobiology, and \\ ${ }^{4}$ Minnesota Veterinary Diagnostic Laboratory, College of Veterinary Medicine, \\ University of Minnesota, St Paul 55108 \\ ${ }^{3}$ School of Public Health, University of Minnesota, Minneapolis 55455
}

\begin{abstract}
The objective of this study was to characterize the distribution of Mycobacterium paratuberculosis (Map) in the environment of infected and uninfected Minnesota dairy farms. Eighty herds known to be infected from Minnesota's Johne's Disease Control Program (JDCP) and 28 herds known to be uninfected from Minnesota Voluntary Johne's Disease Herd Status Program (VJDHSP) were sampled. Fecal samples from up to 100 cows in each herd were cultured in pools of 5 cows. Two environmental samples were obtained from each farm from various locations. All samples were tested using bacterial culture for Map. Eighty percent of the JDCP herds had at least one positive pool. Environmental samples were cultured positive in $78 \%$ of the JDCP herds. Two (7\%) of the VJDHSP herds had one positive pool, and one herd had one positive environmental sample. Environmental samples were cultured positive in cow alleyways (77\% of the herds), manure storage (68\%), calving area $(21 \%)$, sick cow pen (18\%), water runoff $(6 \%)$, and postweaned calves areas (3\%). There was an association between maximum level of colonies per tube from cow alleyways and manure storage and fecal pool prevalence. Herds with both areas cultured negative were estimated to have 0.3 to $4 \%$ fecal pool prevalence. Herds with both areas having a heavy load of bacteria were estimated to have 53 to $73 \%$ fecal pool prevalence. The study results indicate that targeted sampling of cow alleyways and manure storage areas appears to be an alternative strategy for herd screening and Johne's infection status assessment and for estimating herd fecal prevalence.
\end{abstract}

(Key words: Johne's disease, Mycobacterium paratuberculosis, environment, dairy)

Received September 2, 2003.

Accepted June 1, 2004.

Corresponding author: E. A. Raizman; e-mail: raizm001@ umn.edu.

\begin{abstract}
Abbreviation key: CPT = colonies per tube, $\mathbf{J D}=$ Johne's Disease, JDCP = JD Control Program, Map = Mycobacterium paratuberculosis, $\mathbf{M B A H}=$ Minnesota Board of Animal Health, VJDHSP = Voluntary JD Herd Status Program.
\end{abstract}

\section{INTRODUCTION}

Paratuberculosis or Johne's disease (JD) is a chronic and progressive intestinal disease in ruminants caused by Mycobacterium avium spp. paratuberculosis (Map). The usual route of infection is fecal-oral, with young cattle becoming infected by exposure to manure from infected adult cattle or their environment (Larsen et al., 1975; Sweeney, 1996). The disease manifests in adult cows and results in economic losses caused by premature culling, reduced milk production, and loss of BW in cattle sold for slaughter.

Although Map does not propagate in the environment, it survives for long periods in different environmental conditions. Several reports describe long-term Map survival under various in vitro conditions, which include water, urine, manure, and below freezing temperatures (Vishnevskii et al., 1940; Lovel et al., 1944; Larsen et al., 1956; Jørgensen, 1977). These findings suggest that Map survives well in conditions expected on many dairy farms. Currently, not much information is available regarding the distribution of Map in dairy cattle environments. Whitlock et al. (1992) sampled the environment in 11 infected herds in Pennsylvania in which obvious manure samples were not sampled. Those researchers found that $45 \%$ of the farms had positive environmental samples, and only one farm had a high prevalence of environmental contamination (22\%). Similarly, Pavlik et al. (2002) evaluated 2906 environmental samples from 20 infected herds and found that $2 \%$ tested positive for Map.

Johne's disease control programs have been developed in different countries (Kalis et al., 2000; Kennedy and Allworth, 2000) and in several states in the US (Bulaga, 1998) with the goal of testing and classifying 
herds of cattle as infected or presumptively noninfected with maximum accuracy and least cost. Generally, these programs use recognized laboratory tests such as ELISA or direct microbiological individual fecal culture. However, these tests have several disadvantages, especially when applied in herds with subclinical disease or low prevalence. A pooled fecal culture method, which aggregates several cow's fecal samples to one culture unit, has been recently suggested as a good alternative strategy for lowering the procedure cost in herd screening programs for dairy cattle (Kalis et al., 2000a; Wells et al., 2003); however, this still requires individual cattle sampling and has a variable sensitivity, depending on the phase of infection and level of fecal shedding. Because Map is shed into the environment by dairy cattle through fecal contamination and appears to survive well, a better understanding of Map distribution in the environment could lead to improved herd screening alternatives.

The objectives of this study were 1) to describe Map distribution and prevalence in the environment on Minnesota dairy farms and 2) to assess the relationship between culture status of Map in the farm environment and fecal-pooled culture status on dairy farms.

\section{MATERIALS AND METHODS}

\section{Herd Selection and Sampling}

Herds were selected from the database available for 2 JD programs in Minnesota; herds known to be infected from previous testing in the JD Control Program (JDCP) of the Minnesota Board of Animal Health (MBAH) and herds known to be uninfected based on previous testing in the Voluntary JD Herd Status Program (VJDHSP) of the MBAH (Level 1 to 4). Herds were eligible for the study if they were at least $1 \mathrm{yr}$ on the same premise. Two hundred sixteen letters $(75 \%$ from JDCP and 25\% from VJDHSP) were sent to all eligible herd owners to request voluntary participation in the study. Following mailing, herd owners were contacted by phone to confirm their participation in the study.

Participating Minnesota dairy herds were sampled from May to September 2002. From each herd, up to 100 cows were sampled using systematic sampling whenever possible to represent the distribution of cows within each herd. In the free stall housing herds where cows were grouped based on their lactation stage, a proportional number of cows from each pen was sampled. From each cow, approximately $30 \mathrm{~g}$ of fecal material were obtained via rectal retrieval with a plastic disposable rectal examination glove lubricated with sterile water. A new glove was used for each cow sampled. Samples were placed in a $95-\mathrm{mL}$ plastic-covered specimen container and stored in a cooler with ice during transport to the laboratory.

From each study farm, environmental samples were obtained from each of the following locations, if available, on the farm: calving area, dry cow area, cow alleyways, manure storage area, soil from crop fields near cow open areas (dry lot or pasture) where drainage from cow areas to fields was possible, edge of streams where cows have access, water runoff from the parlor or cow barn, preweaned calves area, postweaned calves housing, and sick cow pen. Two samples were collected from each location, with the following exceptions. In preweaned calf areas where a small number of calves were held, only one sample was obtained. Samples were obtained from the calves bedding or hatch walls; however, when not available, the samples were obtained rectally, and each sample contained fecal material from one to 3 calves. In small sick cow pens, only one sample was collected. Each sample contained approximately 20 $\mathrm{g}$ of fecal material with bedding or soil from 3 to 4 different sites within each sampling location. The material was collected with a separate disposable latex glove, placed in a 95-mL plastic-covered specimen container and stored in a cooler with ice during transport to the laboratory.

In free stall barns ( 62 farms), each cow alleyway sample was obtained from 3 to 4 sites across one to 2 alleyways. In tie stall barns (46 farms), samples from cow alleyways were obtained from the gutter on each side of the alleyway. The manure storage lagoon was sampled at 3 to 4 locations at the perimeter edge of the lagoon by submerging the sampling container up to $10 \mathrm{~cm}$ beneath the water surface. Each sample from manure piles was obtained from 3 to 4 different sites up to $10 \mathrm{~cm}$ beneath surface. Each sample from the manure pit was collected using 2 to 3 sterile $4 \times 4$ gauze pads tied to fishing line with fishing weight and soaked at least 10 to $15 \mathrm{~cm}$ below the manure surface. In 2 herds, closed slurries were not sampled for safety reasons. Samples from postweaned calves were obtained from the floor of calf housing areas.

The samples from water stream edges included fecal material in contact with the water and moist soil. Samples of water runoff from the parlor or barn contained moist soil or sediment collected from the bottom of the stream, including water. Samples from fields near cow open areas contained soil from locations where drainage from the cow area was possible.

Herd size information for the study herds and nonparticipant herds was available from JD risk assessments (MBAH, 2003) performed by MBAH and a questionnaire presented to all herd owners by the authors. Average herd 305-d milk production (kg per lactation) reported by the herd owner was available from the 
MBAH risk assessments for 95 study herds and 90 nonrespondent herds.

\section{Laboratory Testing}

Fecal samples from cows were pooled in groups of 5 cows per pool based on age for a total of $10 \mathrm{~g}$ per pool. The procedure involved mixing $2 \mathrm{~g}$ of manure from each of the 5 cows with a wooden stick and thereafter weighing $2 \mathrm{~g}$ for the pooled fecal culture. Fecal pools and environmental samples were tested using bacterial culture for Map at the Minnesota Veterinary Diagnostic Laboratory using the method previously described (Wells et al., 2002a). Briefly, a sedimentation culture procedure was used (Whipple et al., 1991) with $72 \mathrm{~h}$ of sedimentation prior to inoculation of 4 tubes containing Herrold's egg yolk medium. Colony counts were recorded on a weekly basis for $16 \mathrm{wk}$, and final results were scored as negative, light (mean of 0.25 to 9 colonies per tube), moderate (mean of 10 to 49 colonies per tube), and heavy (mean of $\geq 50$ colonies per tube) fecal shedders. Herd or farm environment was defined as infected if at least one pool or environmental sample, respectively, was positive to Map, assuming that the fecal culture method has $100 \%$ specificity.

\section{Statistical Analysis}

Descriptive statistics were used to describe the distribution of the environmental samples using SAS (SAS, 2001). An Independent $t$-test with statistical level $P<$ 0.05 was used to compare herd size and milk production differences between the study herds and nonrespondant herds. A $\chi^{2}$ Fisher exact test with statistical level $P<0.05$ was used to determine the association between environment sampling results and fecal pool status at the herd level. To assess the correlation between the number of positive fecal pools and different positive environment samples, Spearman rank order correlation was applied. The association between fecal pool prevalence and the percentage of different environment locations testing positive was assessed by categorizing the fecal pool prevalence $(<10 \%, 10$ to $30 \%$, and $>30 \%$ ) and use of the $\chi^{2}$ test of association. To determine the best environmental sampling strategy to assess herd infection status, a multivariable logistic regression model was developed using SAS PROC LOGISTIC, where the dependent variable was herd status based on bacterial culture of fecal pools (positive or negative), and the independent variables were environment culture status by location. A forward-selection procedure was used to fit the model, where variables that met the univariable cutoff of $P \leq 0.1$ were fit into the model. To estimate the prevalence of infection in the herd based on environmental sampling areas identified in the selected logistic regression model, the herd prevalence results (percentage of positive fecal pools) were graphed, identifying the beta distribution as the best description of the data distribution. Because some assumptions of ordinary least square models (such as normal distribution and observation independency) would be violated in the data using SAS PROC GLM for the ordinary least sqaures model, PROC MIXED with the EMPIRICAL option was used to fit the model for herd fecal pool prevalence, which enabled us to fit a maximum likelihood Gaussian model with robust standard errors (White, 1980, 1982; Long and Ervin, 2000). This model is robust to misspecification (nonlinear relationship between the dependent and independent variables, lack of observation independency, the omission of a causal variable in the model) and violation of normality. The dependent variable was percent positive fecal pools, and the independent variables were maximum colonies per tube (CPT) by environment location sampled (negative, light, moderate, and heavy), number of positive samples $(0,1,2)$ from the selected farms areas, herd size $(<100,100$ to $200,>200)$, and housing type (free stall or tie stall).

\section{RESULTS}

One hundred thirty-three farm owners $(62 \%)$ confirmed their participation in the study $(63 \%$ of 161 JDCP herds and 56\% of the 55 VJDHSP herds sent cards or responded via a phone call). Twenty-five of the herds ( 22 of the JDCP and 3 of the VJDHSP), which confirmed their participation by letter, were not included in the study for various reasons (i.e., declined participation upon phone call).

One hundred eight herds were sampled including 80 herds from the JDCP and 28 herds from the VJDHSP. At the time of the herd sampling 7, 36, 21, and $36 \%$ of the VJDHSP study herds were at levels $1,2,3$, and 4, respectively. Level 1 VJDHSP study herds were $14 \%$ of the VJDHSP Level 1 herds $(n=14)$, Level 2 study herds were $45 \%$ of the Level 2 VJDHSP herds $(n=22)$, and Level 3 and 4 study herds were 38 and $67 \%$ of the Level $3(\mathrm{n}=16)$ and $4(\mathrm{n}=15)$ VJDHSP herds, respectively. A total of 8645 cows were sampled. and 1739 fecal pools were tested using bacterial culture. In $50 \%$ of the herds, all cows were sampled, whereas an average of $47 \%$ of the cows were sampled in the rest of the 54 herds (minimum, 7\%; maximum, 96\%). The cow breed in the study herds was predominantly Holstein; one herd was predominantly Jersey, and one herd was predominantly Brown Swiss. Nonrespondent herds and herds that were dropped from the study did not statisti- 
Table 1. Study and nonstudy herd descriptive information.

\begin{tabular}{|c|c|c|c|c|}
\hline & $\begin{array}{l}\text { JDCP study herds } \\
(\mathrm{n}=80)\end{array}$ & $\begin{array}{l}\text { Nonparticipant } \\
\text { JDCP herds } \\
(\mathrm{n}=81)\end{array}$ & $\begin{array}{l}\text { VJDHSP study herds } \\
(\mathrm{n}=28)\end{array}$ & $\begin{array}{l}\text { Nonparticipant } \\
\text { VJDHSP herds } \\
(\mathrm{n}=27)\end{array}$ \\
\hline $\begin{array}{l}\text { Herd size average } \\
\text { (minimum; maximum) }\end{array}$ & $217(12 ; 1350)$ & $294(53 ; 1500)$ & $57(20 ; 110)$ & $58(25 ; 100)$ \\
\hline Free stall housing, $\%$ & 76 & $\mathrm{NI}^{1}$ & 7 & $\mathrm{NI}$ \\
\hline Tie stall housing, \% & 24 & NI & 93 & NI \\
\hline $\begin{array}{l}\text { Average milk production, } \\
\text { kg/yr (minimum; maximum) }\end{array}$ & $10,770(6456 ; 12,200)$ & $9953^{2}(5545 ; 17,607)$ & $9965(6760 ; 12,210)$ & $9919^{2}(8182 ; 11,364)$ \\
\hline
\end{tabular}

cally $(P>0.05)$ differ from the study herds in terms of herd size and milk production (Table 1).

Sixty-four (80\%) of the 80 JDCP herds had at least one positive pool; 16 did not have any positive pools. Whole herd sampling was performed in 9 of the 16 JDCP herds with all negative pools, whereas in the remaining 7 herds, partial herd sampling was performed. In these herds, herd size varied between 110 and 1350 milking cows.

Twenty-six of the VJDHSP herds $(n=28)$ were found to be test-negative, with no positive pools; 2 herds had one positive pool each, with a maximum level of shedding of 10 to $50 \mathrm{CPT}$ in one herd and >100 CPT in the other.

The environment around the JDCP farms was found to be contaminated for 61 of the 64 herds with positive fecal pools and in one of the 16 herds with negative pools. In the environmental positive herd with no positive pools, only partial sampling occurred (100 of 210 cows). The environment among the VJDHSP herds was found to be contaminated in one herd (cow alleyways and manure spreader); this herd had a one positive fecal pool as well. A highly significant relationship was found between the environment and fecal pool culture status (odds ratio $=636 ; 95 \% \mathrm{CI}=69$ to $5890 ; P<$ 0.0001). The most common areas found to be contaminated on the farms were cow alleyways (77\% of herds with positive pools or environment) and manure storage (68\%; Table 2). The maximum CPT positive environmental samples on farms was moderate or low (including cow alleyways and manure storage); the maximum CPT for the pooled fecal cultures was high (Figure 1).

Both cow alleyways and manure storage were sampled in $70(88 \%)$ of the JDCP farms, and $56 \%$ of these farms were positive in both areas. At least one sample from the cow alleyway or/and manure storage was Mappositive in $90 \%$ of the herds with positive environment or pools.

Heavily contaminated samples (>50 CPT) from cow alleyways and manure storage areas were found only in herds with high pool prevalence ( $>30 \%$ positive pools). In herds with $>30 \%$ positive pools, all cow alley-

Table 2. Distribution of environmental samples among dairy herds with culture positive pool or environment.

\begin{tabular}{|c|c|c|c|c|c|}
\hline Area on the farm & $\begin{array}{l}\text { Samples } \\
\text { (no.) }\end{array}$ & $\begin{array}{l}\text { Positive } \\
(\%)\end{array}$ & $\begin{array}{l}\text { Farms } \\
\text { tested } \\
\text { (no.) }\end{array}$ & $\begin{array}{l}\text { Farms } \\
\text { positive } \\
(\%)\end{array}$ & $\begin{array}{l}\text { Distribution of } \\
\text { positive farms }\end{array}$ \\
\hline Cows alleyway & 122 & 70 & 66 & 77 & \multirow{6}{*}{$\begin{array}{l}86 \% \text { (Free stall) } \\
14 \% \text { (Tie stall) } \\
98 \% \text { (Calving pe } \\
2 \% \text { (Calving pen } \\
53 \% \text { (Dry lot) } \\
40 \% \text { (Pasture) } \\
7 \% \text { (Pen) }\end{array}$} \\
\hline Calving area & 108 & 17 & 59 & 24 & \\
\hline Dry cow area & 122 & 19 & 61 & 25 & \\
\hline Sick pen & 38 & 45 & 21 & 57 & \\
\hline Preweaned & 125 & 0 & 63 & 0 & \\
\hline Postweaned & 98 & 3 & 52 & 4 & \\
\hline \multicolumn{6}{|l|}{ Environment } \\
\hline Manure storage & 120 & 55 & 60 & 68 & $\begin{array}{l}\text { 68\% (Lagoon) } \\
15 \% \text { (Manure pit) } \\
10 \% \text { (Manure pile) } \\
7 \% \text { (Manure spreader) }\end{array}$ \\
\hline Water access & 18 & 17 & 13 & 7 & \\
\hline Water runoff & 8 & 38 & 4 & 75 & \\
\hline Soil of crop fields & 30 & 0 & 15 & 0 & \\
\hline
\end{tabular}




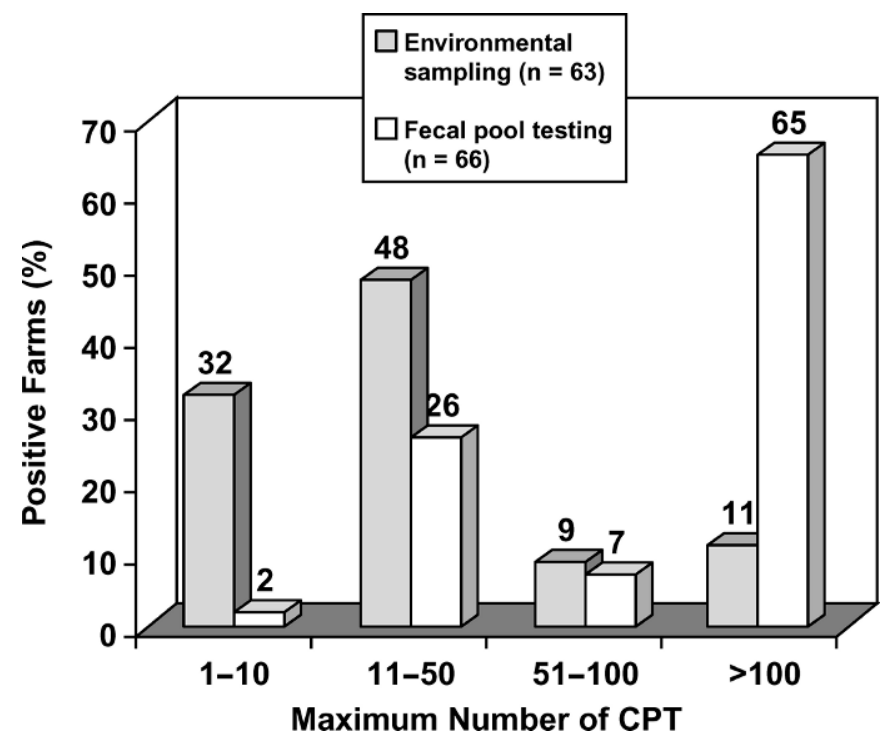

Figure 1. Distribution (\%) of maximum number of colonies per tube (CPT) of environmental and fecal pool culture among positive farms $(\mathrm{n}=67)$.

way samples were positive. Associations were found between the number of farms with positive cow alleyways, manure storage areas, and sick cow pens and the percentage of positive pools (Table 3).

To assess herd infection status (positive vs. negative) based on environmental sampling, the best-fitting logistic regression model included sampling of cow alleyways and manure storage $(-2$ likelihood $=42.72)$, and no other environmental sample contributed additional information to the model (i.e., improved the fit of the model).

The best-fitting model to predict within-herd fecal pool prevalence included the maximum CPT from cow alleyways and manure storage area (Tables 4 and 5). Because of the presence of collinearity, interaction terms were not estimable. Given the model, herds with negative cow alleyways and manure storage have $2 \%$

Table 3. Distribution of positive environment samples based on percentage of positive pools.

\begin{tabular}{llcc}
\hline & \multicolumn{3}{c}{ Positive pools (n=67) } \\
\cline { 2 - 4 } Farm housing area & 1 to $10 \%$ & 10 to $30 \%$ & $>30 \%$ \\
\cline { 2 - 4 } & 20 & 30 & 19 \\
Calving area & 36 & 12 & 33 \\
Dry cow area & $59^{\mathrm{a}}$ & $72^{\mathrm{a}}$ & $100^{\mathrm{b}}$ \\
Cows alleyway & $44^{\mathrm{a}}$ & $68^{\mathrm{b}}$ & $85^{\mathrm{b}}$ \\
Manure storage & $29^{\mathrm{a}}$ & $57^{\mathrm{a}}$ & $86^{\mathrm{b}}$ \\
Sick pen & 67 & 17 & 33 \\
Water access & 0 & 5 & 5 \\
Postweaned calves & 0 & 5 &
\end{tabular}

${ }^{\mathrm{a}, \mathrm{b}}$ Different superscripts indicate statistical significance $(P<0.05)$.
$(95 \% \mathrm{CI}=0.3$ to $4 \%)$ positive fecal pool prevalence; heavy contamination in both areas results in the highest positive fecal pool prevalence $(63 \%$; $95 \% \mathrm{CI}=53$ to $73 \%)$.

\section{DISCUSSION}

This is the first large-scale study evaluating the distribution of Map in cattle and environment surrounding dairy farms using different types of cattle housing, various herd sizes, and a variety of locations in the farm environment.

Herds were selected to the study based on their voluntary participation in MBAH programs; therefore, it can be assumed that farms that participated in this study have elevated JD awareness. This may explain the positive response rate of $62 \%$. In both MBAH programs, study herds did not statistically differ from nonrespondant herds, indicating that the study population is a good representation of herds that participate in both MBAH JD programs.

One limitation of this study is related to fecal culture sensitivity constraints. In the current study, the limited pool test sensitivity for light shedders (1 to $10 \mathrm{CPT}$ ) might have failed to detect some of the JDCP herds, which are presumed infected. Wells et al. (2002b) found that the sensitivity of detection for Map was greater with a smaller pool size (i.e., 5 vs. 10 samples per pool); the sensitivity was $44 \%$ in pools with one of 5 cows shedding at low levels and was $94 \%$ in pools with one of 5 cows shedding at high levels. Overall, Wells et al. (2003) showed a herd sensitivity of $100 \%$ for high prevalence herds using fecal pools and $94 \%$ for lower prevalence herds using fecal pools. In the current study, presumably, if undetected, these truly infected herds would have a very low prevalence of infection. Because a maximum of 100 cows per herd was sampled in this study, it is possible that infected cows (especially low shedders) were present and were not detected in herds where not all of the cows were sampled. Interestingly, all herds with negative fecal pools, except one, had negative environments as well.

The environmental sampling procedure used in the current study, which involved the collection of 3 to 4 scoops of feces from each location, is a form of a pooling procedure. It is possible, however, that environmental samples containing small numbers of bacteria $(<10$ CPT) may not be detected as positive (as happened in 4 farms that had positive fecal pools but with no positive environmental samples) because it may fall below the sensitivity of the culture procedure. Nevertheless, all herds with high pool prevalence were detected with environmental sampling. 
Table 4. Coefficients from multivariate regression model (robust SE) estimating fecal pool prevalence based on manure storage and cow alleyway environmental sample testing. ${ }^{1}$

\begin{tabular}{llllr}
\hline Effect & Estimate & $\mathrm{SE}$ & $\mathrm{t}$ value & $P>|\mathrm{t}|$ \\
\hline Intercept & 2.1 & 0.9 & 2.3 & 0.022 \\
Light alleyway & 16.3 & 3.3 & 4.9 & $<0.0001$ \\
Moderate alleyway & 17.6 & 4.21 & 4.2 & $<0.0001$ \\
Heavy alleyway & 34.2 & 4.3 & 8.0 & $<0.0001$ \\
Negative alleyway & Reference level & & & \\
Light manure storage & 10.2 & 2.9 & 3.5 & 0.0007 \\
Moderate manure storage & 10.9 & 5.4 & 2.0 & 0.0467 \\
Heavy manure storage & 26.6 & 3.3 & 8.1 & $<0.0001$ \\
Negative manure storage & Reference level & &
\end{tabular}

${ }^{1}-2$ likelihood $=116.7 ;$ Akaike information criteria $=118.7 ; \mathrm{df}=88$.

The US VJDHSP for cattle was designed to identify uninfected or low-risk JD herds (Bulaga, 1998). The testing protocol involves testing a subset of adult cows in a herd using a serum ELISA to detect antibodies against Map and follow-up testing of ELISA-positive cattle by fecal culture to confirm the presence of Map. The first test (Level 1 of 4) of the VJDHSP includes 30 serologic samples per herd. Because the ELISA test sensitivity is low $(15 \%)$ in subclinically infected low fecal shedding cows (Sweeney et al., 1995), the Level 1 test strategy only detects an estimated $33 \%$ of herds with $<5 \%$ prevalence and $68 \%$ of herds with 5 to $9 \%$ prevalence of JD (Wells et al., 2002b). After 2 yr of testing, herds that tested negative in 2 consecutive ELISA tests can progress to Level 3. For Level 3, the test includes fecal cultures on a statistical subset of animals $\geq 3 \mathrm{yr}$ of age. This may explain why in the current study, 2 of the VJDHSP herds, both on Level 2 of the program, tested positive in this study.

The environmental sampling results in this study suggest that the initial assessment of farm infection status does not necessarily require individual cattle serologic testing, which has limitations because of its low sensitivity, especially in low shedding subclinically infected cows, and because it requires handling of individual cattle. The results of the current study suggest that assessment of herd infection status can be performed by targeted sampling of the cow alleyway and manure storage areas. This approach suggests a conve- nient and promising alternative strategy for herd screening and JD infection status assessment and could potentially replace the first screening ELISA tests for Level 1 of the VJDHSP or for JDCP herds that were never previously tested.

The basic test result information can provide further applications. The statistical models developed in this study offer a practical way to estimate the herd fecal pool apparent prevalence by using the maximum CPT of cow alleyways and manure storage. Individual cow prevalence can also be estimated from fecal pool prevalence (Cowling et al., 1999; Evers and Nauta, 2001). Sampling cow alleyways and manure storage has the potential of saving significant economical resources in terms of cost and time relative to individual animal testing. Based on this approach, it is estimated that 30 min of sampling and $\$ 100$ (US) laboratory fees are required to estimate herd infection status with reasonable (approximately 90\%) accuracy. Achieving the same goal using ELISA test requires at least $\$ 180$ (US) laboratory fees in addition to veterinary labor fees to sample individual cattle. A few herds had negative environment samples and positive fecal pools, suggesting a higher sensitivity of fecal pool culture over the environmental sampling. Therefore, it is suggested that more advanced testing in the VJDHSP include fecal pool culture of cows, which has been shown to be more sensitive and cost effective than serum ELISA (Wells et al., 2003).

Table 5. Estimation of fecal pool prevalence (with 95\% CI) based on maximum shedding level of 2 samples from each of 2 environmental locations $(\mathrm{n}=60)$.

\begin{tabular}{llllll}
\hline & & & Cow alleyway & & \\
\cline { 2 - 5 } & $\begin{array}{l}\text { Maximum level } \\
\text { of shedding }\end{array}$ & Negative & Light & Moderate & Heavy \\
\hline \multirow{3}{*}{ Manure storage } & Negative & $2 \%(0.3$ to $4 \%)$ & $18 \%(12$ to $24 \%)$ & $20 \%(12$ to $28 \%)$ & $36 \%(27$ to $45 \%)$ \\
& Light & $12 \%(7$ to $17 \%)$ & $29 \%(22$ to $36 \%)$ & $30 \%(22$ to $39 \%)$ & $46 \%(38$ to $55 \%)$ \\
& Moderate & $13 \%(3$ to $23 \%)$ & $29 \%(16$ to $42 \%)$ & $31 \%(21$ to $39 \%)$ & $47 \%(36$ to $58 \%)$ \\
& Heavy & $29 \%(23$ to $35 \%)$ & $45 \%(36$ to $57 \%)$ & $46 \%(36$ to $56 \%)$ & $63 \%(53$ to $73 \%)$ \\
\hline
\end{tabular}


The presence of Map in manure storage is a serious concern among farms that spread manure on crop fields that are later used as silage and hay for feeding cattle. This practice was previously described as an important risk factor for within-herd transmission of infection (Obasanjo et al., 1997). In the current study, many herds spread manure on their crop fields (data not presented). Although all samples obtained from soil of crop fields were cultured negative, it is important to consider the small number of farms where fields were sampled (14\%) and the probability of Map detection with only 2 samples from a vast area such as corn or alfalfa fields.

In the current study, samples from all preweaned calves were negative for Map, but positive samples from postweaned calves (4 to 6 mo of age) were found in 2 herds. Sweeney et al. (1992) found that ingestion of 55 $\times 10^{5} \mathrm{cfu}$ was enough to detect 36 to $68 \mathrm{CPT}$ passively shed in feces. Therefore, passive shedding because of gastrointestinal transit and subsequent contamination from adult cattle manure should be considered, as well as active shedding, as possible explanation for positive results. Map shedding in young animals was described only in experimentally infected calves that were inoculated with the bacteria via nasogastric tube (MacDonald et al., 1999), feed milk (Collins, 1994), orally (Larsen et al., 1975; Larsen and Miller, 1977), subcutaneously, and intravenously (Taylor, 1953; Larsen and Miller, 1977). Because Map can be isolated from the feces of uninfected cattle subsequent to ingestion of feces from infected cows (Sweeney et al., 1992), the isolation of the organism from cattle feces on farms with heavy environmental contamination should be interpreted with caution (Sweeney et al., 1992). Regardless of whether the contamination in the postweaned calf area is due to external contamination through utensils, boots, etc. or due to active shedding, the presence of the bacteria in the area of young heifers is a risk for Map transmission in young heifers (4 to 6 mo of age) housed in groups (Collins, 1994; Wells and Wagner, 2000).

Finally, because the study was performed during the summer and only in Minnesota, and herd sampling and infection status assessment is needed year around across the country, it is important to obtain further information about Map distribution, especially during the winter months and in different parts of the US and worldwide.

\section{CONCLUSIONS}

The association between infected herds and their infected environment emphasizes the critical importance of farm management strategies for the control of JD to reduce environmental Map contamination and exposure to cattle.

Targeted common and contaminated areas in the farm environment, specifically cow alleyways and manure storage (especially lagoons), suggest a promising alternative strategy for herd screening and JD infection status assessment and for estimating herd fecal prevalence. This strategy has the potential of saving significant economical resources in terms of cost and time.

\section{ACKNOWLEDGMENTS}

The authors want to acknowledge the Minnesota Rapid Agricultural Response Fund and the Minnesota Board of Animal Health for providing funding for this study and to thank and appreciate the assistance of: all the dairy herds owners that participated in this study and their staff, Minnesota Veterinary Diagnostic Laboratory, Kirk Mueller and Brian Rose for their field assistance, and Norma Velasquez for her long comprehensive support.

\section{REFERENCES}

Bulaga, L. L. 1998. US Voluntary Johne's Disease Herd Status Program for cattle. Pages 420-433 in Proc. 102nd Annu. Mtg. US Anim Health Assoc., Minneapolis, MN. Animal Health Assoc., Richmond, VA.

Collins, T. M. 1994. Early diagnosis of $M$. paratuberculosis infections in young cattle. Pages 52-56 in Proc. 15th Tech. Conf. AI Reprod., Milwaukee, WI. The National Assoc. of Anim. Breeders, Columbia, MO.

Cowling, D. W., I. A. Gardner, and W. O. Johnson. 1999. Comparison of methods for estimation of individual level prevalence based on pooled samples. Prev. Vet. Med. 39:211-225.

Evers, E. G., and M. J. Nauta. 2001. Estimation of animal-level prevalence from pooled samples in animal production. Prev. Vet. Med. 49:175-190.

Jørgensen, J. B. 1977. Survival of Mycobacterium paratuberculosis in slurry. Nord. Vet. Med. 29:267-270.

Kalis, C. H. J., H. W. Barkema, and J. W. Hesselink. 2000a. Certification of dairy herds as free paratuberculosis using culture of strategically pooled fecal samples. Page 171 in Proc. 9th Int. Symp. Vet. Epidemiol. Econ. Breckenridge, CO. Int. Soc. Vet. Epidemiology and Economics, Fort Collins, CO.

Kalis, C. H. J., J. W. Hesselink, and H. W. Barkema. 2000. Culture strategically pooled bovine fecal samples as a method to screen herds for paratuberculosis. J. Vet. Diagn. Invest. 12:547-551.

Kennedy, D. J., and M. B. Allworth. 2000. Accepting the challenge of controlling Johne's Disease in Australia. Page 81 in Proc. 9th Int. Symp. Vet. Epidemiol. Econ. Breckenridge, CO. Int. Soc. Vet. Epidemiology and Economics, Fort Collins, CO.

Larsen, A. B., S. Richard, B. S. Merkal, and T. H. Vardman. 1956. Survival time of Mycobacterium paratuberculosis. Am. J. Vet. Res. 17:549-551.

Larsen, A. B., R. S. Merkal, and R. C. Cultip. 1975. Age of cattle related to resistance to infection with Mycobacterium paratuberculosis. Am. J. Vet. Res. 36:255-257.

Larsen, A. B., and J. M. Miller. 1977. Mycobacterium paratuberculosis compared with intravenous and oral exposures. Am. J. Vet. Res. 38:1669-1671.

Long, J. S., and L. H. Ervin. 2000. Using Heteroscedasticity consistent standard errors in the Linear Regression Model. Am. Stat. $54: 217-224$. 
Lovel, R., M. Levi, and J. Francis. 1944. Studies on the survival of Johne's bacilli. J. Comp. Pathol. 54:120-129.

MacDonald, W. L., S. E. Ridge, A. F. Hope, and R. J. Condron. 1999. Evaluation of diagnostic tests for Johne's Disease in young cattle. Aust. Vet. J. 77:113-119.

Minnesota Board of Animal Health (MBAH). 2003. Subject: Johne's disease Risk Assessment. http://www.bah.state.mn.us/diseases/ johnes/mn_johnes.htm. Accessed May 2004.

Obasanjo, I. O., Y. T. Gröhn, and H. O. Mohammed. 1997. Factors associated with the presence of Mycobacterium paratuberculosis infection in dairy herds on the New York state paratuberculosis control program. Prev. Vet. Med. 32:243-251.

Pavlik, I., A. W. Yayo, O. Fischer, L. Maltova, P. Svastova, M. Bartos, M. Machackova, M. Alex, and J. Lamka. 2002. Role of the external environment, plants and non-vertebrate for the spread of $\mathrm{Myco}$ bacterium avium subsp paratuberculosis. Page 514 in Proc. 7th Int. Colloquium on Paratuberculosis, Bilbao, Spain. Int. Assoc. of Paratuberculosis, Inc., Madison, WI.

SAS/STAT User's Guide (Release 8.02). 2001. SAS Inst. Inc., Cary, $\mathrm{NC}$

Sweeney, R. W., R. H. Whitlock, C. L. Buckley, and P. A. Spencer. 1995. Evaluation of a commercial enzyme-linked immunosorbant assay for the diagnosis of paratuberculosis in dairy cattle. J. Vet. Diagn. Invest. 7:488-493.

Sweeney, R. W., R. H. Whitlock, A. N. Hamir, A. E. Rosenberger, and S. A. Herr. 1992. Isolation of Mycobacterium paratuberculosis after oral inoculation in uninfected cattle. Am. J. Vet. Res. 53:1312-1314.

Sweeney, R. W. 1996. Transmission of paratuberculosis. Vet. Clin. North Am. 12:305-312.

Taylor, A. W. 1953. Experimental Johne's Disease in cattle. J. Comp. Pathol. 63:355-367.
Vishnevskii, P. P., E. G. Manatsev, V. V. Chernyshev, and N. S. Ustoichivost. 1940. Paratuberkuliznykh batsill Ione. Sovyet Vet. 11-12:89-93.

Wells, S. J., and B. A. Wagner. 2000. Herd level risk factors for infection with Mycobacterium paratuberculosis in US dairies and association between familiarity of the herd manager with disease or prior diagnosis of the disease in that herd and use of preventive measures. JAVMA 216:1450-1457.

Wells, S. J., R. H. Whitlock, C. J. Lindeman, and T. Fyock. 2002a Evaluation of bacteriologic culture of pooled fecal samples for detection of Mycobacterium paratuberculosis. Am. J. Vet. Res. 63:1207-1211.

Wells, S. J., R. H. Whitlock, and B. A. Wagner. 2002b. Sensitivity of test strategies used in the Voluntary Johne's Disease Herd Status Program for detection of Mycobacterium paratuberculosis infection in dairy cattle herds. JAVMA 220:1053-1057.

Wells, S. J., S. M. Godden, C. J. Lindman, and J. E. Collins. 2003. Evaluation of bacteriologic culture of individual and pooled fecal samples for detection of Mycobacterium paratuberculosis in dairy cattle herds. JAVMA 223:1022-1025.

White, H. 1980. A Heteroskedacity-consistent covariance matrix estimator and a direct test of Heteroskedacity. Econometrica 48:817-838.

White, H. 1982. Maximum likelihood estimation for misspecified models. Econometrica 50:1-26.

Whipple, D. L., D. R. Callihan, and J. L. Jarnagin. 1991. Cultivation of Mycobacterium paratuberculosis from bovine fecal specimens and a suggested standardized procedure. J. Vet. Diagn. Invest. 3:368-373.

Whitlock, R. H., A. E. Rosenberger, M. Siebert, and R. Sweeney. 1992. Environment contamination of dairy farms with cattle infected with Mycobacterium paratuberculosis. Proc. XVII World Buiatrics Congr., St Paul, MN 1:84. 